OPEN ACCESS

Edited by:

C. Wang

Wuhan University, China

Reviewed by:

Marc Iglarz,

Actelion Pharmaceuticals, Switzerland

Gaetano Santulli,

Columbia University, USA

Ali Hussein Eid,

American University of Beirut,

Lebanon

Yuanyuan $\mathrm{Li}$,

Northeast Ohio Medical University,

USA

*Correspondence:

Yuan Lin

363978171@qq.com

tThese authors have contributed equally to this work.

Specialty section:

This article was submitted to Cardiovascular and Smooth Muscle

Pharmacology,

a section of the journal

Frontiers in Pharmacology

Received: 28 June 2016 Accepted: 01 November 2016

Published: 25 November 2016

Citation:

Chen D, Lv B, Kobayashi S, Xiong Y, Sun $P$, Lin $Y$, Genovese $S$, Epifano $F$, Hou S, Tang F, Ji Y and Yu D (2016) Madagascine Induces Vasodilatation via Activation of AMPK.

Front. Pharmacol. 7:435. doi: 10.3389/fphar.2016.00435

\section{Madagascine Induces Vasodilatation via Activation of AMPK}

\author{
Dapeng Chen ${ }^{1 \dagger}$, Bochao Lv't, Sei Kobayashi' ${ }^{2}$, Yongjian Xiong ${ }^{3}$, Pengyuan Sun ${ }^{1}$, \\ Yuan Lin ${ }^{1 *}$, Salvatore Genovese ${ }^{4}$, Francesco Epifano ${ }^{4}$, Shanshan Hou' ${ }^{1}$, Fusheng Tang ${ }^{1}$, \\ Yunyan $\mathrm{Ji}^{1}$ and Dandan $\mathrm{Yu}^{1}$
}

${ }^{1}$ Dalian Medical University, Dalian, China, ${ }^{2}$ Yamaguchi University School of Medicine, Yamaguchi, Japan, ${ }^{3}$ Central Laboratory, the First Affiliated Hospital, Dalian Medical University, Dalian, China, ${ }^{4}$ Dipartimento di Farmacia, Università degli Studi "G. D'Annunzio" Chieti-Pescara, Chieti, Italy

Madagascine (3-isopentenyloxyemodin) can be chemically synthesized or purified from several Rhamnus species, and it is found to have more potent biological activities than the parent compound emodin. The aim of this study is to characterize the vasodilatory effect of madagascine on vasoconstriction and sphingosylphosphorylcholine induced vasospasm in ex vivo and reveal the potential mechanisms in vitro. The effects of madagascine on vasoconstriction of rat mesenteric resistance arteries (MRAs) induced by $\mathrm{K}^{+}$, methoxamine, and endothelin-1 were, respectively, studied. The cholesterol-enriched porcine coronary vascular smooth muscle (VSM) strips were used to investigate the effects of madagascine on abnormal constriction induced by sphingosylphosphorylcholine (SPC) which has a pivotal role in vasospasm. The vasodilatory effect was induced by madagascine $(0.3-100 \mu \mathrm{M})$ in isolated rat MRAs and the vasodilatory effect was blocked by NO synthase inhibitor L-NAME and AMPK inhibitor compound C. Madagascine $(10 \mu \mathrm{M})$ also significantly relaxed the abnormal constriction in porcine VSM induced by SPC and the effect was abolished by compound C. Madagascine significantly increased the phosphorylation of endothelial nitric oxide synthase (eNOS) in endothelial cells while decreasing the phosphorylation of myosin phosphatase target subunit 1 (MYPT1) in VSM cells. Madagascine-induced vasodilatation was abrogated using small interfering RNA knockdown of AMPK. In summary, madagascine exerted vasodilatation through activating AMPK, leading to the activation of eNOS in endothelium and inhibition of ROCK/MYPT1 in VSM. This study suggests the potential value of madagascine in amelioration of vasospasm related cardiovascular diseases.

Keywords: madagascine, AMPK, eNOS, vasospasm, vasoconstriction

\section{INTRODUCTION}

Vascular vasodilatation is beneficial for amelioration of cardiovascular diseases including essentialand renal-parenchymal-disease-related hypertension, vascular remodeling, cardiac infarction, and congestive heart failure (Hisham and Bayraktutan, 2012; Kumar et al., 2012; Machino et al., 2014). Vascular tone is determined by the vasoconstriction and is regulated by a complex interplay of vasodilator and vasoconstrictor substances (Wang et al., 2011). The drugs which can directly or indirectly induce vascular vasodilatation including inhibitors of renin-angiotensin 
system, antagonists of adrenergic receptors, diuretics, nitrates, calcium channel blockers. (Kondo et al., 1979; Cox and Rusch, 2002; Ji, 2013; Salihi, 2013) possess the potential in ameliorating cardiovascular diseases.

Recent studies suggest AMP-activated protein kinase (AMPK) is a new therapeutic target for vasodilatation. In endothelial cells, AMPK is activated through the phosphorylation by LKB1 and CAMKK (Stahmann et al., 2006). The activation of AMPK leads to vasodilatation through the phosphorylation of epithelial nitric oxide synthase (eNOS) at site Ser1177 in epithelium and a direct inhibition of vascular smooth muscle (VSM) constriction (Reihill et al., 2007; Bradley et al., 2010; Shuangxi et al., 2011). Smooth muscle constriction is induced by phosphorylation of 20-kDa myosin light chain (MLC), which is regulated by both $\mathrm{Ca}^{2+}$ dependent and $\mathrm{Ca}^{2+}$ independent mechanisms (Somlyo and Somlyo, 1994). AMPK activation leads to inhibition of Rho-associated protein kinase (ROCK) which mediates $\mathrm{Ca}^{2+}$ independent VSM constriction by inhibiting myosin phosphatase via phosphorylation of myosin phosphatase target subunit 1 (MYPT1) (Somlyo, 2002; Shuangxi et al., 2011). Sphingosylphosphorylcholine (SPC) generated by $N$-deacylation of sphingomyelin which is one of the most abundant lipids in cell membrane (Yue et al., 2015). SPC is a phospholipid mediator in blood plasma and it exert multifunctional role in cell physiological regulation (Satoshi et al., 2002). SPCmediated activation of ROCK has been proved to be involved in pathogenesis of vasospasm (Fumiaki et al., 2002; Somlyo, 2002). However, the relationship and between AMPK activation and SPC induced vasospasm remains unknown.

Madagascine (3-isopentenyloxyemodin) is a natural compound containing an anthraquinone core linked to a 3, 3-dimethylallyoxy chain (Figure 1). The trivial name of madagascine derives from the natural source, Harungana madagascariensis Poir, from which it was isolated and structurally characterized for the first time (Ritchie and Taylor, 1964; Epifano et al., 2013). Subsequently madagascine has also been obtained from several other natural sources including medicinal plants belonging to Rhamnus spp. (Delle Monache et al., 1987; Iinuma et al., 1995; Mbaveng et al., 2008). Natural compounds containing an anthracene are widely distributed in the plant kingdom and madagascine emerged as one of the most promising compound from a pharmacological point of view (Epifano et al., 2013). Madagascine have been reported to have multiple biological activities including antioxidant, antimicrobial, and anticancer effects (Mbaveng et al., 2008; Ee et al., 2011; Epifano et al., 2013).

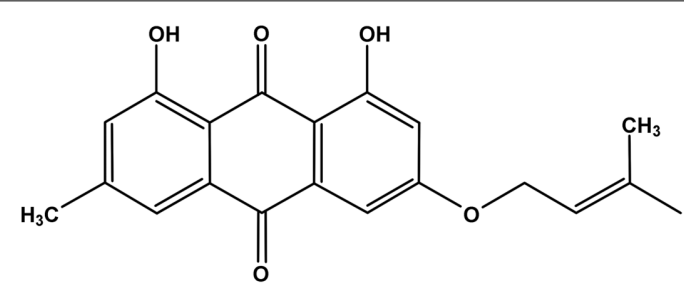

FIGURE 1 | Chemical structure of madagascine.
Emodin is found to induce the activation of AMPK in skeletal muscle and liver cells (Song et al., 2013; Subramaniam et al., 2013). Under the same experimental conditions, compared with emodin, the biological activity was more potent and safe (Mbaveng et al., 2008; Ee et al., 2011). Based on our preexperiments, the present study was designed to characterize the vasodilatory effect of madagascine on vasoconstriction and SPC induced vasospasm in ex vivo and in vitro. The isolated rat mesenteric resistance arteries (MRAs) were used to investigate the effects of madagascine-mediated activation of AMPK on vasoconstriction. The porcine coronary arteries were used to investigate the effects of madagascine-mediated activation of AMPK on abnormal constriction induced by SPC because porcine coronary arteries are cholesterol-enriched and cholesterol potentiates the $\mathrm{Ca}^{2+}$ independent of VSM constriction mediated by SPC (Noriyasu et al., 2006). The vasodilator properties of madagascine were also investigated by using human umbilical vein endothelial cells (HUVECs) and human coronary artery smooth muscle cells (HCASMCs), respectively.

\section{MATERIALS AND METHODS}

\section{Animals}

The experimental protocol was approved by Dalian Medical University Animal Care and Ethics Committee at June 8th, 2012, and all animals used were maintained in accordance with National Institutes of Health Guide for Care and Use of Laboratory Animals (Publication no. 85-23, revised 1985). Fifty Male Wistar rats (weighing 200-300 g) were obtained from Experimental Animal Center, Dalian Medical University. [Certificate of Conformity: No. SCXK (Liao) 2008-0002]. The animal protocol was designed to minimize pain or discomfort to the animals. The animals were acclimatized to laboratory conditions $\left(23^{\circ} \mathrm{C}, 12 \mathrm{~h} / 12 \mathrm{~h}\right.$ light/dark, 50\% humidity, ad libitum access to food and water) for 2 weeks before experimentation. All rats were euthanized by barbiturate overdose (intravenous injection, $150 \mathrm{mg} / \mathrm{kg}$ ) for mesenteric arteries isolation. Porcine coronary arteries $(20-30 \mathrm{~mm}$ from the origin of the proximal portion of left anterior descending arteries) were obtained from a local abattoir.

\section{Reagents and Cell Lines}

Madagascine was supplied by Francesco Epifano and Salvatore Genovese. AMPK- $\alpha 1+\alpha 2$ antibodies (ab800039), AMPK- $\alpha 1$ (phospho T183) + $\alpha 2$ (phospho T172) antibodies (ab72845), eNOS antibodies (ab5589), and eNOS (phospho S1177) antibodies (ab184154) were bought from Abcam (Hong Kong) Ltd. (Hong Kong, China). MYPT1 p-MYPT1 (phospho Ser 695) antibodies (sc-33360) were bought from Santa Cruz Biotechnology, Inc. (Santa Cruz, CA, USA). MLC antibodies (3672) and phosphor-MLC (Ser 19) antibodies (3671) were bought form Cell Signaling Technology, Inc. (USA). GAPDH (10494-1-AP) antibodies and MYPT1 antibodies (22117-1-AP) were bought form Proteintech Group, Inc. (Wuhan, China). SPC was bought from Biomol. Unless otherwise indicated, chemicals 
were obtained from Sigma-Aldrich (St Louis, MO, USA). The cell HUVECs and HCASMCs were obtained from cell bank of Shanghai Institute (Shanghai, China). The cells used in this study were evaluated before experiment including the expression of eNOS and AMPK in these cell lines. No significant inter-species variations in AMPK and eNOS signaling which affect the results in this study were observed according to previous publications and pre-experiments.

\section{Perfusion of Rat Mesenteric Resistance Arteries}

The rat MRAs were isolated and prepared for perfusion according to the methods by Sun et al. (2009). The MRAs were placed in organ bath maintained at $37^{\circ} \mathrm{C}$, perfused with a modified Krebs solution [modified Krebs solution in mM: $\mathrm{NaCl} \mathrm{119.0,}$ $\mathrm{KCl}$ 4.7, $\mathrm{CaCl} 2$ 2.4, $\mathrm{MgSO} 4$ 1.2, $\mathrm{NaHCO} 3$ 25.0, $\mathrm{KH} 2 \mathrm{PO} 4$
1.2, EDTA 0.03, and D-glucose $11.1(\mathrm{pH} \mathrm{7.4)]} \mathrm{at} \mathrm{a} \mathrm{constant}$ flow rate of $5 \mathrm{~mL} / \mathrm{min}$ with a peristaltic pump (Chengdu TME Technology Co, Ltd, China). Changes in the perfusion pressure were recorded by BL-420F biological system (Chengdu TME Technology Co, Ltd, China) (Chen et al., 2015). The endothelium of the MRAs was removed through the perfusion of $1.80 \mathrm{mg} / \mathrm{mL}$ sodium deoxycholate in saline for $30 \mathrm{~s}$ as described previously (Shiraki et al., 2000). The MRAs were perfused with $100 \mathrm{mM}$ papaverine (PPV) to induce complete relaxation for confirmation of the vascular activity at the end of each experiment.

\section{Simultaneous Measurement of $\left[\mathrm{Ca}^{2+}\right] \mathrm{i}$ and Force of Porcine VSM In situ}

Porcine coronary arteries were placed in $4^{\circ} \mathrm{C}$ physiological salt solution (PSS; in mM: $123 \mathrm{NaCl}, 4.7 \mathrm{KCl}, 15.5 \mathrm{NaHCO}_{3}, 1.2$
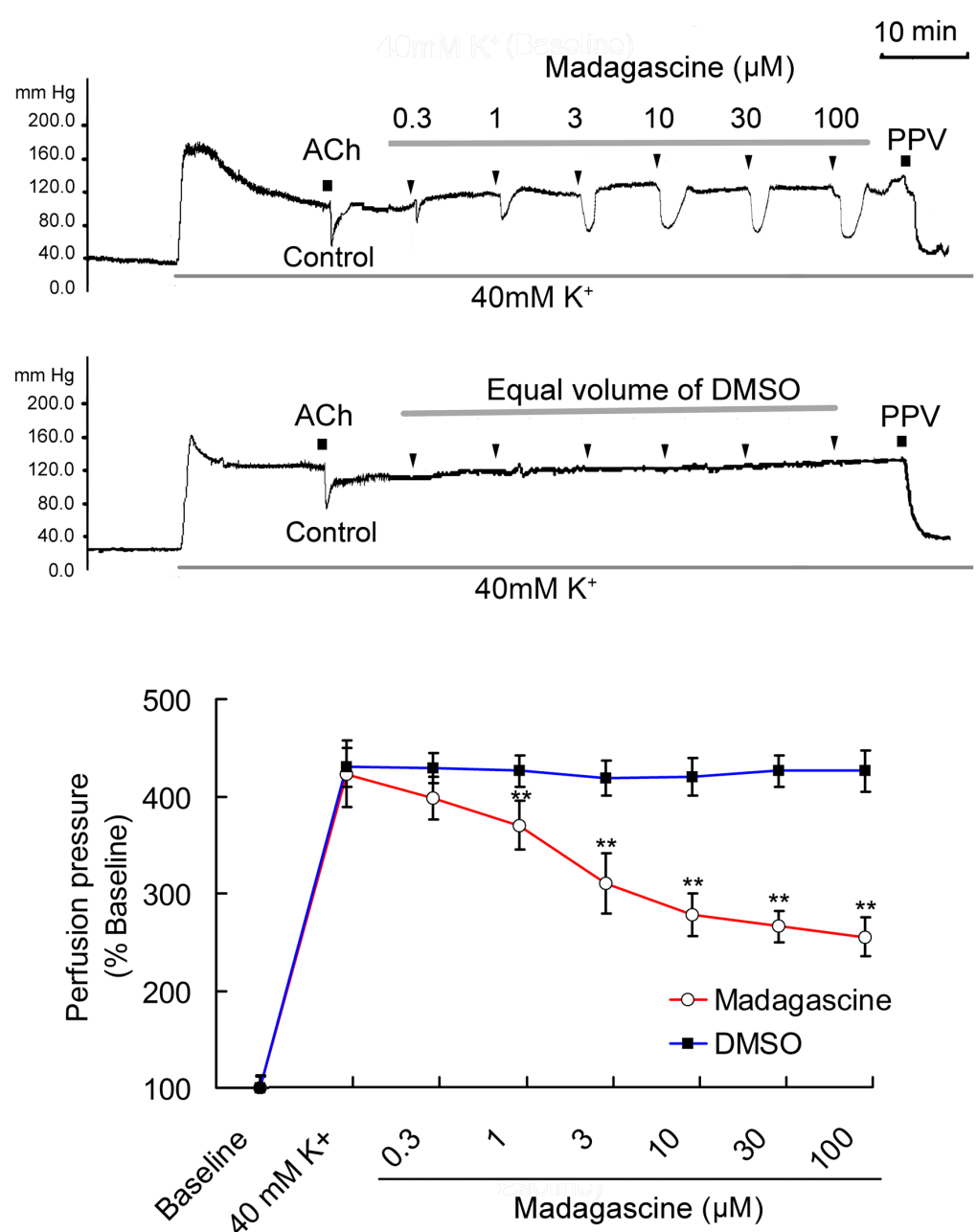

FIGURE 2 | Madagascine (0.3-100 $\mu \mathrm{M}$ ) inhibited the constriction of rat mesenteric resistance arteries (MRAs) induced by $\mathbf{4 0}$ mM $\mathbf{K}^{+}$. The doses of madagascine were selected according to pre-experiments. Isolated rat MRAs with intact endothelium were perfused with Krebs solution and the perfusion pressure was stimulated by continuous perfusion of $40 \mathrm{mM} \mathrm{K}^{+}$. After the elevated perfusion pressure stabilized, Krebs solution containing 40 mM K ${ }^{+}$and madagascine at a concentration of $0.3,1,3,10,30$, or $100 \mu \mathrm{M}$ was perfused, respectively. Acetylcholine (ACh, $1 \mathrm{nM}$ ) treatment is a bolus injection. Vasodilatation response to ACh confirms the intact of endothelium. Data are expressed as the mean $\pm \mathrm{SD}$ and the response to Krebs solution was set as baseline. Other data are the relative values compared with baseline. ${ }^{* *} p<0.01$ compared with $40 \mathrm{mM} \mathrm{K}^{+}, n=6$ tissues. 


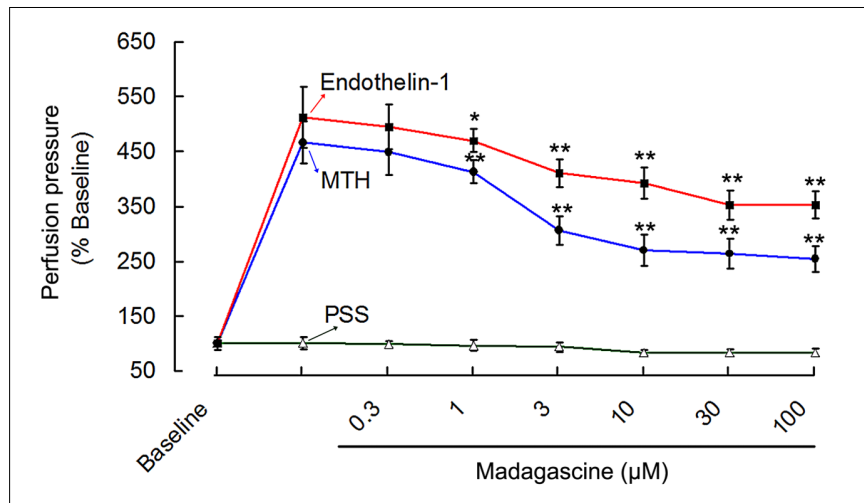

FIGURE 3 | Madagascine (0.3-100 $\mu \mathrm{M})$ inhibited the constriction of rat MRAs induced by endothelin-1 (10 nM) and methoxamine (MTH,

$7 \mu \mathbf{M})$. The perfusion pressure of isolated rat MRAs with intact endothelium were stimulated by continuous perfusion of endothelin-1/methoxamine, respectively. After the elevated perfusion pressure stabilized, Krebs solution containing endothelin-1/methoxamine and madagascine at a concentration of $0.3,1,3,10,30$, or $100 \mu \mathrm{M}$ was perfused, respectively. The intact of endothelium was confirmed through vasodilatation response to ACh. Data are expressed as the mean \pm SD and the response to Krebs solution was set as baseline. Other data are the relative values compared with baseline.

${ }^{*} p<0.05,{ }^{* *} p<0.01$ compared with endothelin-1/methoxamine, $n=6$ tissues.

$\mathrm{KH}_{2} \mathrm{PO}_{4}, 1.2 \mathrm{MgCl}_{2}, 1.25 \mathrm{CaCl}_{2}$, and $11.5 \mathrm{D}$-glucose). The arteries were cut into strips $(1 \mathrm{~mm} \times 4 \mathrm{~mm})$ without endothelium and adventitia. These strips were mounted vertically at the organ bath filled with PSS, gassed with $5 \% \mathrm{CO}_{2} / 95 \% \mathrm{O}_{2}$, and maintained at $37^{\circ} \mathrm{C}$. The isometric force of VSM strips was measured by a force transducer (TB-612T, Nihon Koden). Effect of madagascine on contractile force was investigated at the maximum and steady state of SPC or $40 \mathrm{mM} \mathrm{K}^{+}$induced constriction.

The contractile force and changes in intracellular $\mathrm{Ca}^{2+}$ $\left(\left[\mathrm{Ca}^{2+}\right] \mathrm{i}\right)$ were simultaneously measured using porcine VSM strips (Fumiaki et al., 2002). The VSM strips were loaded with $12.5 \mu \mathrm{M}$ fura-2/AM. Changes in $\left[\mathrm{Ca}^{2+}\right] \mathrm{i}$ were continuously recorded with a spectrofluorometer (CAM-230, Japan Spectroscopic) equipped with a randomized optical fiber system (Todoroki-Ikeda et al., 2000; Noriyasu et al., 2006).

\section{Western Blot Analysis}

Total protein was isolated from HUVECs or HCASMCs. The blots on nitrocellulose filter membrane were probed with corresponding antibodies. The bands were detected and quantified using MultiSpectral imaging system (UVP, Cambridge, $\mathrm{UK})$.

\section{Cell Transfection}

The HUVECs or HCASMCs cells were transfected with Lipofectamine 2000 (Invitrogen) and AMPK- $\alpha 1 / \alpha 2$-targeted or a control small interfering RNA (siRNA) oligos (Dharmacon, Lafayette, CO, USA) according to the manufacturer's instructions (Takara Biotechnology (Dalian) CO., LTD). The siRNA sequence was as follows: 50-ACC GAG CUA UGA AGC AGC UGG GUU U-30. The efficiency of gene silencing was confirmed by Western blotting analysis.
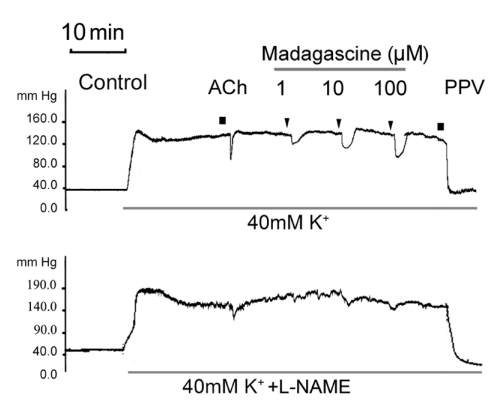

Endothelium

intact
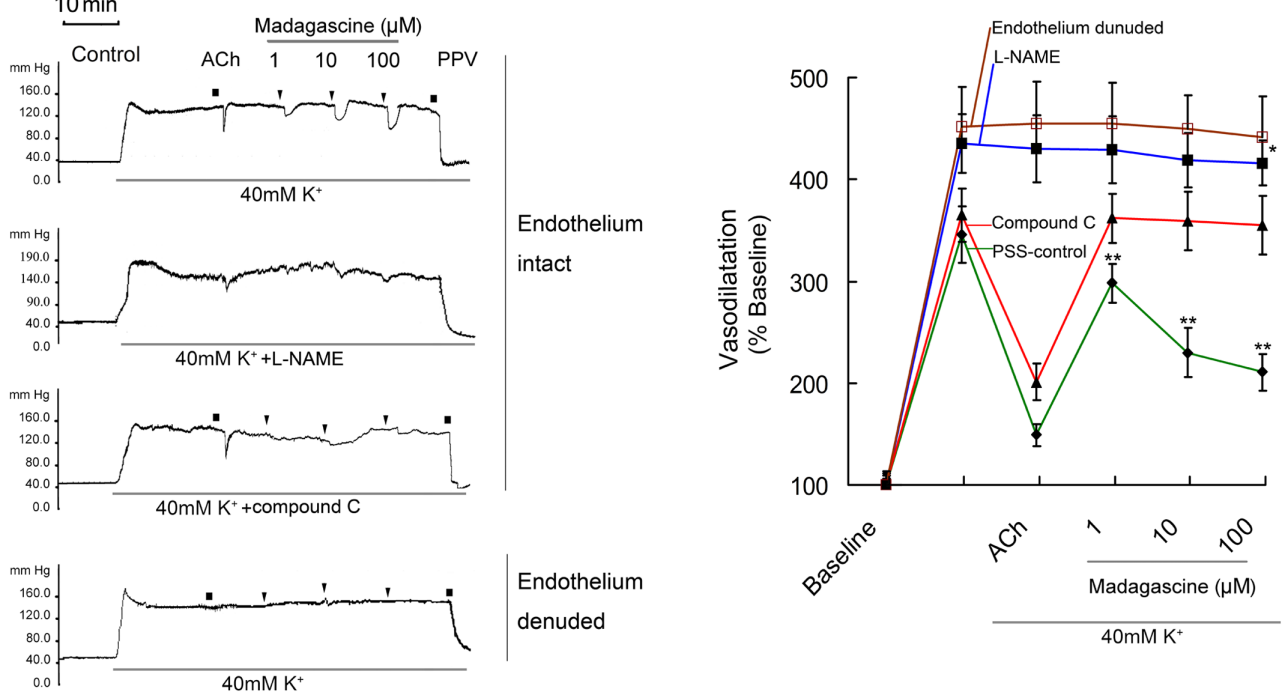

FIGURE 4 | Effects of compound $\mathbf{C}$ and L-NAME on madagascine-exerted vasodilatation. After $40 \mathrm{mM} \mathrm{K} \mathrm{K}^{+}$induced high perfusion pressure stabilized, Krebs solution containing $40 \mathrm{mM} \mathrm{K}^{+}+\mathrm{L}-\mathrm{NAME}(100 \mu \mathrm{M})$ and $40 \mathrm{mM} \mathrm{K}^{+}+$compound $\mathrm{C}(20 \mu \mathrm{M})$ was perfused, respectively. And after the perfusion pressure stabilized, Krebs solution containing $40 \mathrm{mM} \mathrm{K}^{+}+\mathrm{L}-\mathrm{NAME}(120 \mu \mathrm{M})+$ madagascine and $40 \mathrm{mM} \mathrm{K}+$ + compound $\mathrm{C}(20 \mu \mathrm{M})+$ madagascine was perfused. Acetylcholine (ACh, $1 \mathrm{nM}$ ) treatment is a bolus injection. Vasodilatation response to ACh confirms the intact of endothelium. Data are expressed as the mean $\pm \mathrm{SD}$ and the response to Krebs solution was set as baseline. Other data are the relative values compared with baseline. ${ }^{*} p<0.05$ compared with stable elevated perfusion pressure by Krebs solution containing both $40 \mathrm{mM} \mathrm{K}^{+}$and L-NAME, $n=6$ tissues; ${ }^{* *} p<0.01$ compared with stable elevated perfusion pressure by Krebs solution containing $40 \mathrm{mM} \mathrm{K}^{+}$(PSS control), $n=6$ tissues. 


\section{Statistical Analysis}

Data analysis was conducted in a blinded manner according to single-blind study design. The One-Way ANOVA was used where three or more groups of data were compared. Data were expressed as the mean $\pm \mathrm{SD}$. The data followed a normal distribution and each group had equal variances. To further evaluate the data, Kruskal-Wallis rank sum test was used. All experiments were repeated for at least six times.

\section{RESULTS}

\section{Effects of Madagascine on the Vasoconstriction of MRAs}

Based on pre-experiments, $0.3-100 \mu \mathrm{M}$ madagascine were selected to study its vasodilatory effects. Madagascine reduced constriction of rat isolated MRAs induced by $40 \mathrm{mM} \mathrm{K} \mathrm{K}^{+}$ in a concentration range of 0.3-100 $\mu \mathrm{M}$. Madagascineinduced vasodilatation was transient and disappeared within about $6 \mathrm{~min}$. Equal volume of vehicle DMSO did not show any inhibitory effect on the constriction of MRAs (Figure 2). Madagascine also relaxed MRAs pre-contracted by methoxamine and endothelin-1. However, madagascine did not significantly change the basal tension (Figure 3). The rat MRAs remained in good condition after madagascine treatment by washing.

\section{Effects of L-NAME and Compound C on Madagascine Induced Vasodilatation}

Madagascine did not induced vasodilatation on $40 \mathrm{mM}$ $\mathrm{K}^{+}$-induced constriction of rat MRAs without epithelium
(Figure 4). In MRAs with an intact endothelium, madagascine exerted vasodilatation effect was significantly blocked by NO synthase inhibitor L-NAME and AMPK inhibitor compound C, respectively. These results suggest that AMPK/eNOS signaling pathway is involved in madagascine-induced vasodilatation (Figure 4). Both AMPK/eNOS and AMPK/AKT/eNOS are known to be related to increase the production of NO (Bradley et al., 2010; Wei et al., 2016). As shown in Figure 5, madagascineinduced vasodilatation in the presence of SC66 was more transient than that in the absence of SC66. However, in this study, AKT inhibitor did not significantly block madagascine induced vasodilatation rate (Figure 5). The potential role of AMPK/eNOS pathway was mainly studied in this study.

\section{Effects of Madagascine on AMPK and eNOS in HUVECs}

To validate the involvement of AMPK/eNOS signaling pathway in madagascine exerted vasodilatation, the effects of madagascine on AMPK and eNOS were studied using HUVECs. Madagascine, in a concentration range of 0.3$30 \mu \mathrm{M}$, induced the phosphorylation of eNOS and the phosphorylation of AMPK, respectively, in a time dependent manner from 1 to $24 \mathrm{~min}$ (Figure 6). The effects of AMPK knockdown by siRNA in HUVECs were confirmed by Western blotting analysis (Figures 7A,B). After siRNA knockdown of AMPK, the madagascine-induced phosphorylation of eNOS was abrogated, indicating that madagascine-induced phosphorylation of AMPK activated the phosphorylation of eNOS, leading to nitrite-mediated blood vessel relaxation (Figure 7).
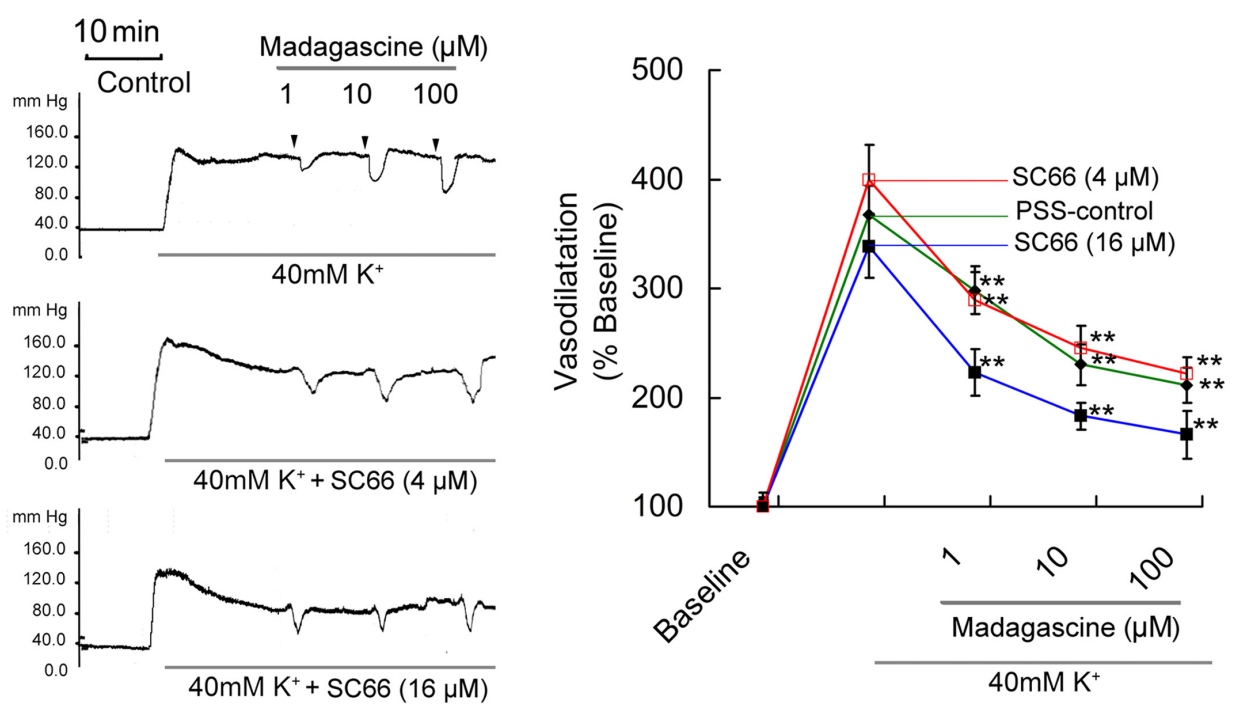

FIGURE 5 | Effects of AKT inhibitor SC66 on madagascine-exerted vasodilatation. After 40 mM $\mathrm{K}^{+}$induced high perfusion pressure stabilized, Krebs solution containing $40 \mathrm{mM} \mathrm{K}^{+}+$SC66 $(4 \mu \mathrm{M})$ and $40 \mathrm{mM} \mathrm{K}^{+}+$SC66 $(16 \mu \mathrm{M})$ was perfused, respectively. And after the perfusion pressure stabilized, Krebs solution containing $40 \mathrm{mM} \mathrm{K}^{+}+$SC66 $(4 \mu \mathrm{M})+$ madagascine and $40 \mathrm{mM} \mathrm{K}{ }^{+}+$SC66 $(16 \mu \mathrm{M})+$ madagascine was perfused. Data are expressed as the mean $\pm \mathrm{SD}$ and the response to Krebs solution was set as baseline. Other data are the relative values compared with baseline. ${ }^{* *} p<0.01$ compared with stable elevated perfusion pressure by Krebs solution containing $40 \mathrm{mM} \mathrm{K}^{+}$and SC66, $n=6$ tissues. 


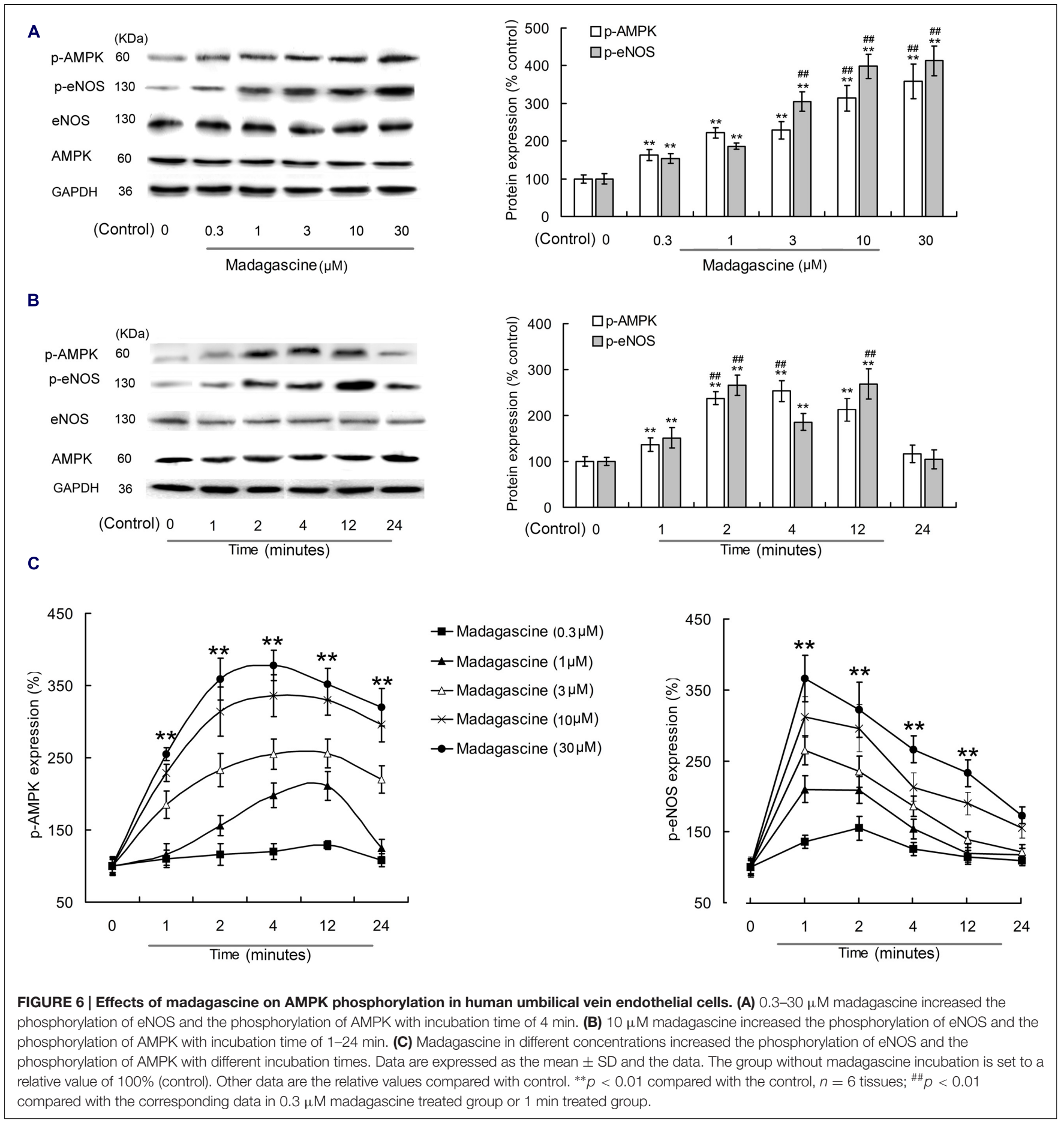

\section{Effects of Madagascine on SPC Induced Abnormal Constriction}

Madagascine did not affect $40 \mathrm{mM} \mathrm{K}{ }^{+}$-induced constriction in rat MRAs without epithelium, suggesting that madagascineactivated AMPK induced vasodilatation of VSM is $\mathrm{Ca}^{2+}$-independent, because $\mathrm{K}^{+}$depolarization induced vasoconstriction is a typical $\mathrm{Ca}^{2+}$ dependent constriction (Satoshi et al., 2002; Somlyo, 2002).
As SPC is found to induce $\mathrm{Ca}^{2+}$ independent vasoconstriction through activation of ROCK which is involved in hypertension and vasospasm (Satoshi et al., 2002), the effects of madagascine on vasospasm induced by SPC were investigated. The porcine coronary arteries were used here because porcine coronary arteries are cholesterol-enriched and cholesterol potentiates the $\mathrm{Ca}^{2+}$ independent of VSM constriction mediated by SPC as described above. As shown in Figure 8A, 


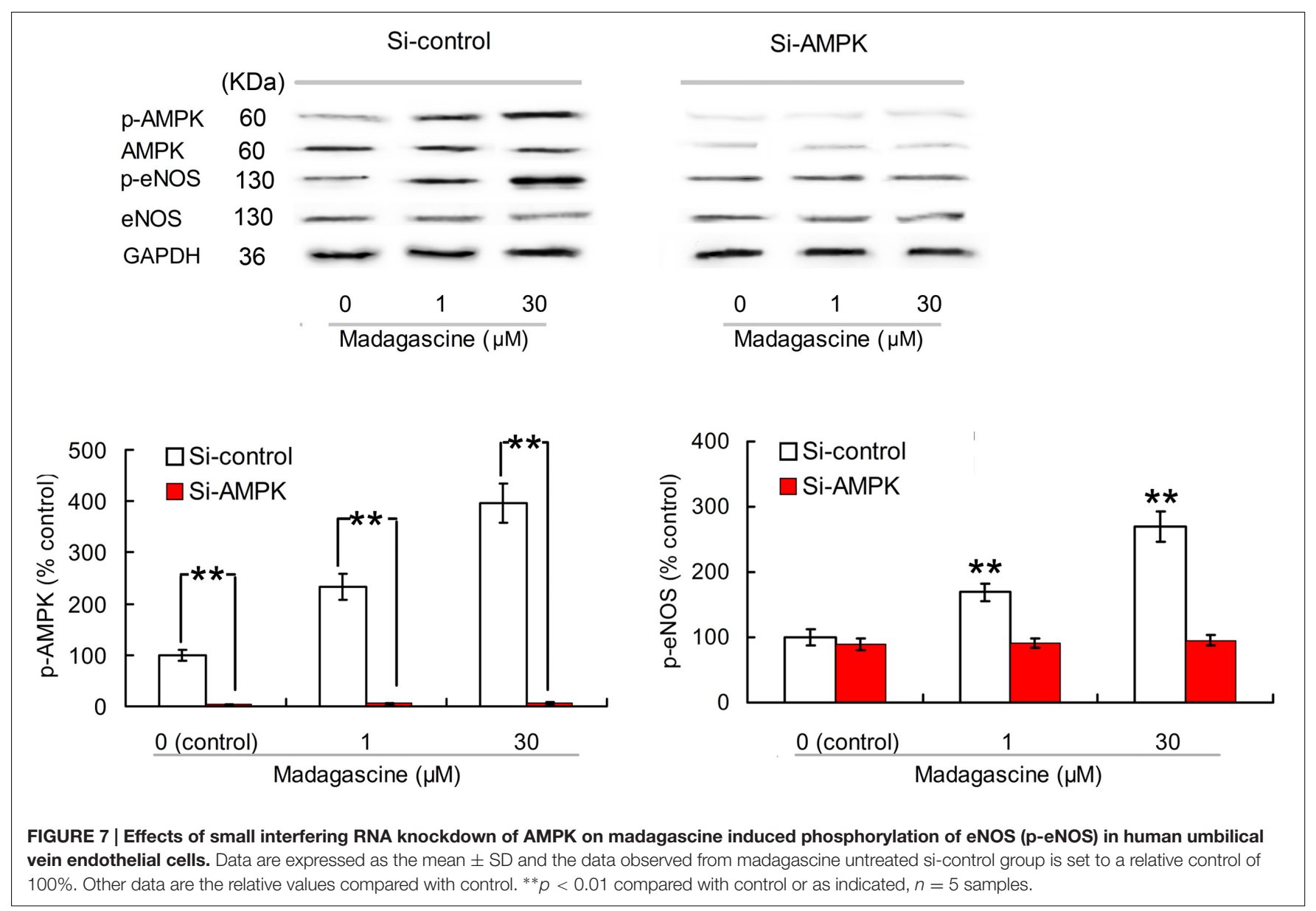

madagascine affects neither the increase of $\left[\mathrm{Ca}^{2+}\right] \mathrm{i}$ nor the constriction of VSM induced by $40 \mathrm{mM} \mathrm{K}^{+}$. However, SPC induced VSM constriction was significantly reduced by madagascine without significant affecting the slightly elevated $\left[\mathrm{Ca}^{2+}\right]$ i (Figure 8B); and madagascine induced vasodilatation was abolished by AMPK inhibitor compound C (Figure 8C), indicating that madagascine reduced SPCinduced $\mathrm{Ca}^{2+}$ independent vasoconstriction via activating AMPK.

\section{Effects of Madagascine on Ampk, Mypt1, and Mlc20 in Hcasmcs}

To validate the involvement of AMPK in madagascinereduced abnormal constriction induced by SPC, the effects of madagascine on the phosphorylation of MYPT1 and phosphorylation of $\mathrm{MLC}_{20}$ in VSM cells with siRNA knockdown of AMPK were studied. The effects of AMPK knockdown by siRNA in HCASMCs were confirmed by Western blotting analysis (Figures 9A,C). The phosphorylation of MYPT1 and the phosphorylation of $\mathrm{MLC}_{20}$ were significantly increased in HCASMCs incubated with SPC (Figures 9A,E,F). Madagascine significantly decreased both SPC-elevated phosphorylation of MYPT1 and the phosphorylation of $\mathrm{MLC}_{20}$. The inhibitory effects of madagascine on the phosphorylation of MYPT1 and the phosphorylation of $\mathrm{MLC}_{20}$ were abrogated by siRNA knockdown of AMPK (Figures 9A,E,F), indicating that madagascine-induced phosphorylation of AMPK inhibited the activity of ROCK (the expression of phosphorylate MYPT1 represents the ROCK activity), leading to the vasodilatation of $\mathrm{Ca}^{2+}$-independent vasoconstriction. In order to prevent the false-positive results by ROCK independent phosphorylation of MYPT1 (Kaplun et al., 2003), ROCK inhibitor Y-27632 was also used. As shown in Figures 9B,D, the inhibitory effects of madagascine on the phosphorylation of MYPT1 were also significantly abrogated by Y-27632, which confirms that madagascine induced activation of AMPK leads to the inhibition of ROCK.

\section{DISCUSSION}

In this study, madagascine exerted vasodilatory effects in rat MRAs and porcine VSM strips, respectively. The phosphorylation of AMPK was significantly increased by madagascine in a concentration and time dependent manner. The phosphorylation of eNOS in HUVECs was significantly increased and the phosphorylation of MYPT1 in HCASMC cells was significantly decreased by madagascine. 


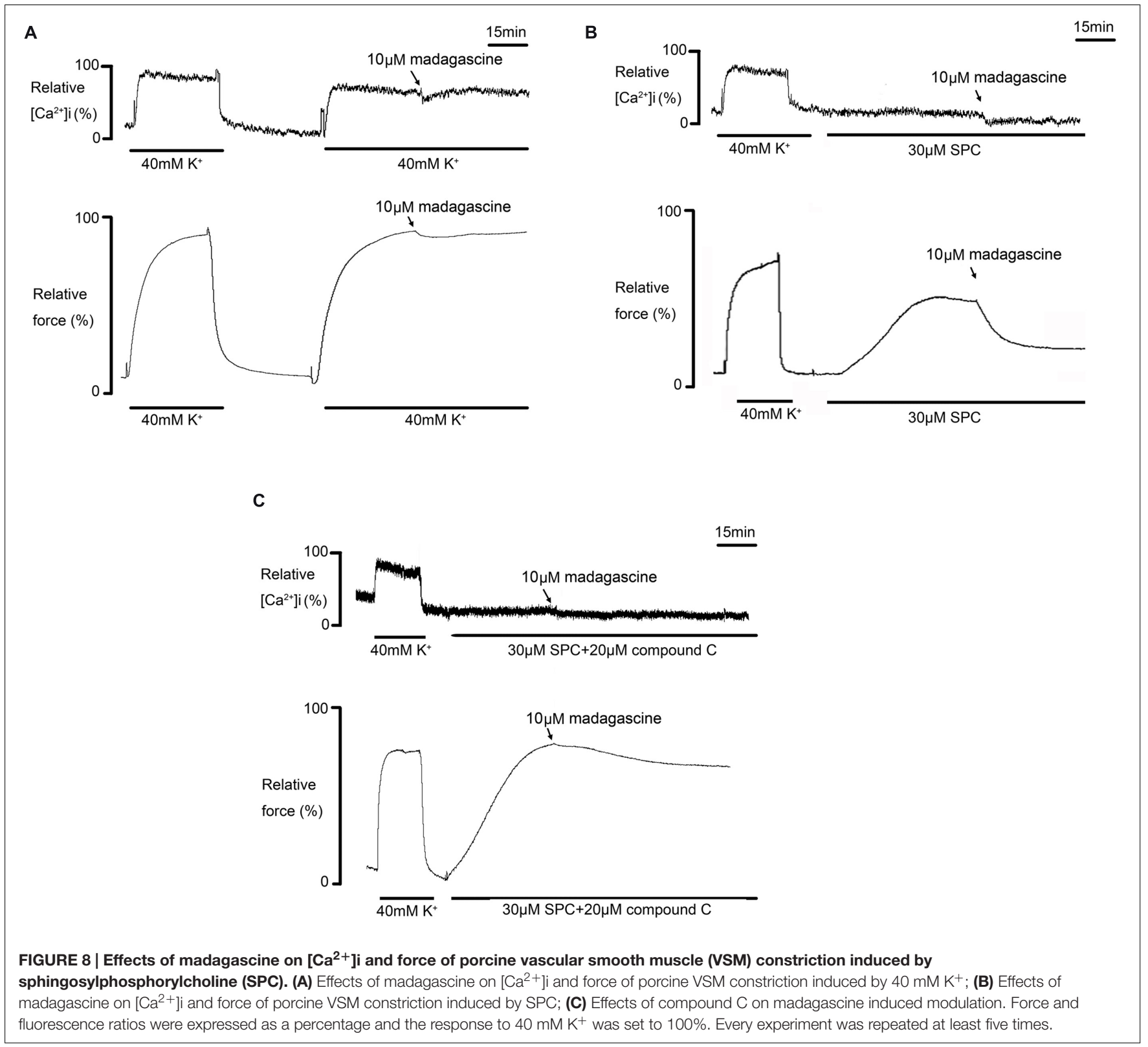

These results suggest that AMPK-mediated activation of eNOS in epithelium and inhibition of ROCK/MYPT1 in VSM were involved in madagascine-induced vasodilatation (Figure 10).

In the vasculature, the activation of endothelial AMPK has been shown to phosphorylate eNOS at $\operatorname{Ser}^{1177}$ and $\operatorname{Ser}^{633}$, stimulating NO release and subsequent vasodilatation of blood vessels (Majithiya and Balaraman, 2006). Purified AMPK is also reported to phosphorylate eNOS at $\mathrm{Thr}^{495}$ in vitro (Mariman and Wang, 2010). In this study, we also found that in the presence of AKT inhibitor SC66, madagascine induced vasodilatation was more transient than that in the absence of SC66. This suggests that the AMPK/AKT/eNOS pathway may also be involved in madagascine induced vasodilatation. AKT pathway may attribute to increase madagascine induced vasodilatation time. However, how can AKT modulate madagascine induced madagascine needs further study.

In this study, madagascine inhibited $40 \mathrm{mM} \mathrm{K} \mathrm{K}^{+}$induced MRAs constriction and the inhibition was blocked by AMPK inhibitor compound $\mathrm{C}$ and eNOS inhibitor L-NAME. These results suggested that AMPK activation in epithelium is involved in madagascine-induced MRAs vasodilatation.

AMPK-mediated relaxation of VSM constriction is also observed where the endothelium is damaged (Majithiya and Balaraman, 2006), suggesting that AMPK activation mediated vasodilatation is not dependent on endothelium. AMPK activation can lead to $\mathrm{Ca}^{2+}$ independent vasoconstriction 
A

$$
\text { (KDa) }
$$

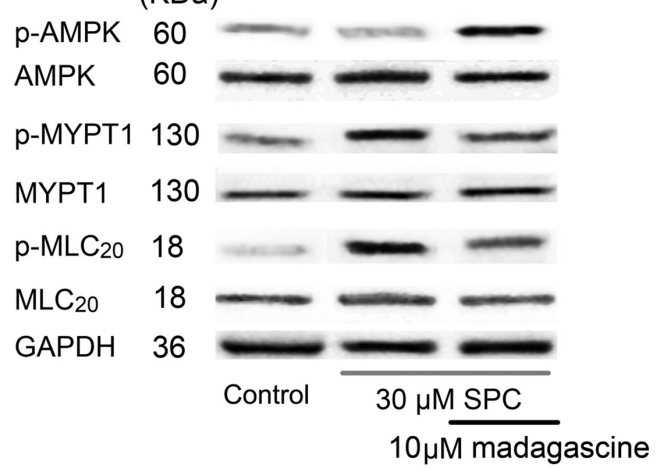

B

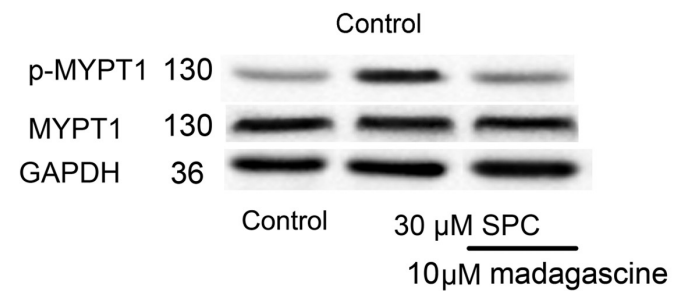

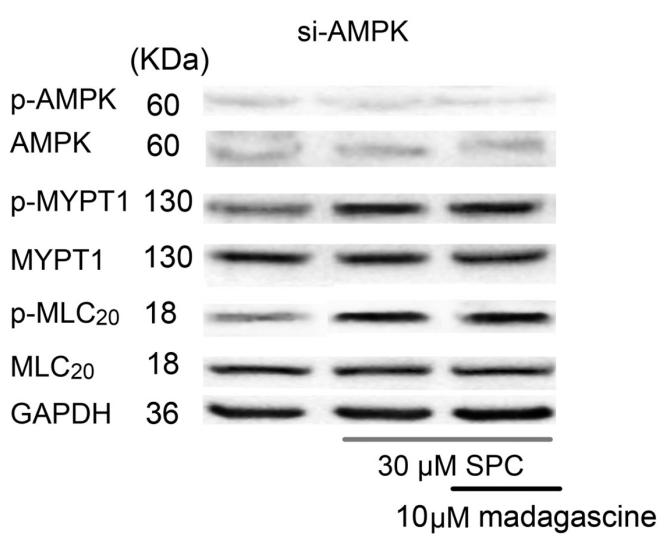

Y-27632

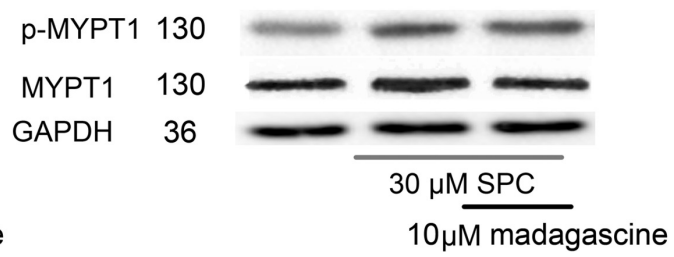

C

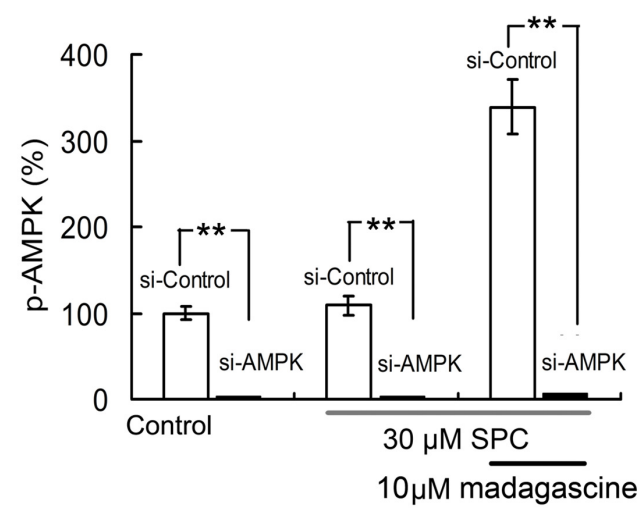

E

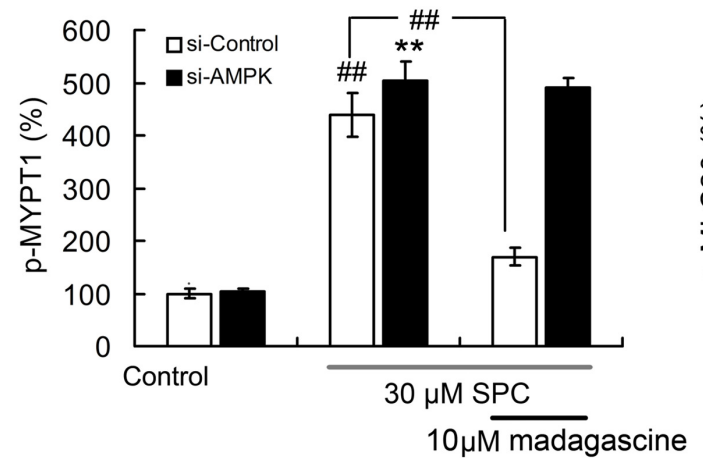

D

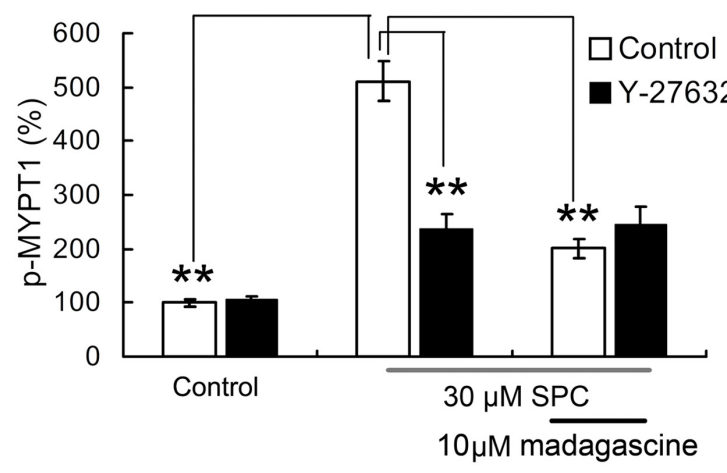

F

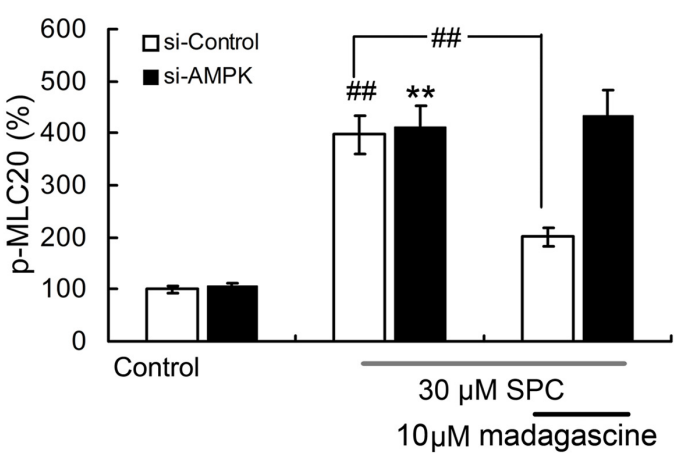

FIGURE 9 | Effects of small interfering RNA (siRNA) knockdown of AMPK and ROCK inhibitor Y27632 (10 $\mu$ M) on madagascine induced the phosphorylation of MYPT1 (p-MYPT1) and the phosphorylation of MLC20 (p-MLC $\left.{ }_{20}\right)$ in human coronary artery smooth muscle cells. (A), (C), (E), and (F): Effects of siRNA knockdown of AMPK on madagascine induced the phosphorylation of AMPK (p-AMPK), p-MYPT1 and p-MLC20. (B) and (D): Effects of Y27632 on madagascine induced p-MYPT1. Data are expressed as the mean \pm SD and the data obtained from madagascine untreated si-control group is set to a relative value of $100 \%$ (control). Other data are the relative values compared with control. ${ }^{* *} p<0.01$ compared with madagascine untreated si-AMPK group or as indicated, $n=6$ samples; ${ }^{\#} p<0.01$ compared with data obtained from madagascine untreated si-control group or as indicated, $n=6$ samples. 


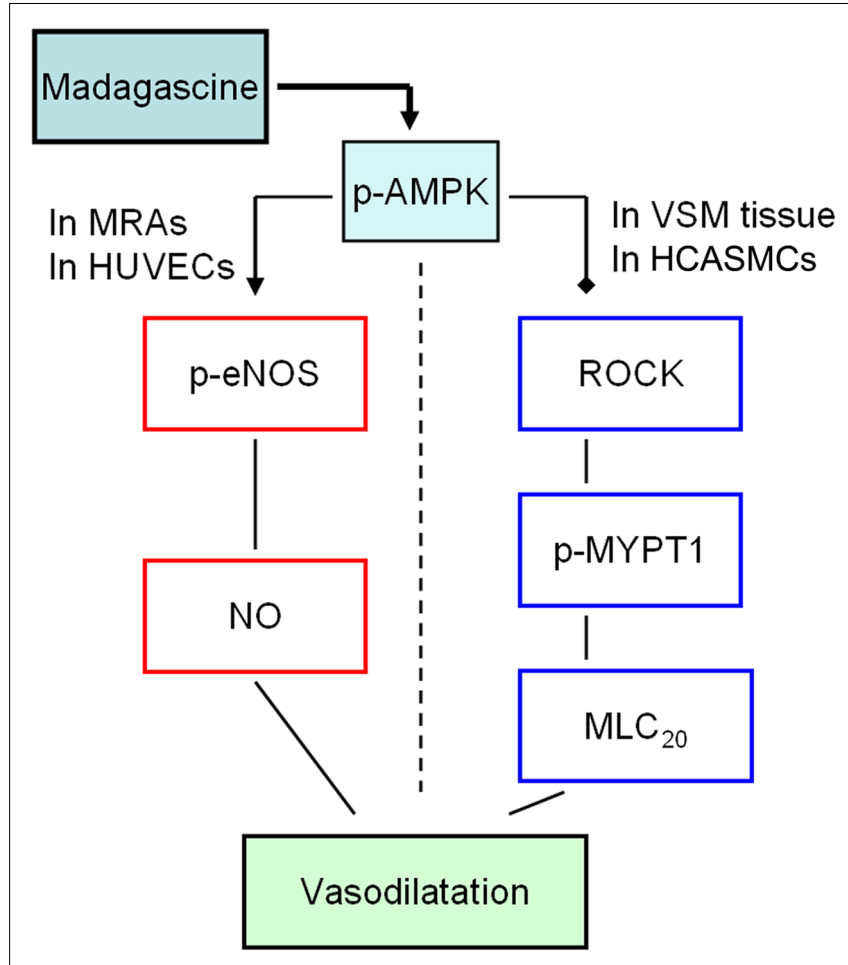

FIGURE 10 | The potential mechanisms involved in madagascine-activated AMPK induced vasodilatation. The solid black squares represent for inhibitory effect; the arrow represents for stimulatory effect. MRAs, mesenteric resistance arteries; VSM, vascular smooth muscle; HUVECs, human umbilical vein endothelial cells; HCASMCs, human coronary artery smooth muscle cells.

(Somlyo, 2002; Shuangxi et al., 2011) and this effect was also confirmed in the present study as madagascine did not show any relaxant effects on the constriction of MRAs without endothelium in the presence of $40 \mathrm{mM} \mathrm{K}^{+}$(data not shown). Madagascine significantly inhibited the VSM constriction induced by SPC and the inhibition was not abolished by compound $\mathrm{C}$, suggesting that $\mathrm{AMPK}$ activation is involved in madagascine induced VSM relaxation. In HCASMC cells, SPC-induced increase in the phosphorylation of MYPT1 and the phosphorylation of $\mathrm{MLC}_{20}$ were significantly reversed by madagascine. AMPK knock down block madagascineinduced the inhibition of the phosphorylation of MYPT1 (represents for the ROCK activity) and the phosphorylation of $\mathrm{MLC}_{20}$. The results suggest that madagascine-induced AMPK activation is beneficial for amelioration of vasospasm as SPC-mediated activation of ROCK is found to be involved in pathogenesis of vasospasm (Fumiaki et al., 2002; Somlyo, 2002). Although it is applicable to test the drug effects on AMPK or eNOS using HUVEC and HCASMCs (Fumiaki et al., 2002; Satoshi et al., 2002; Noriyasu et al., 2006; Yang et al., 2009; Xu et al., 2012), it is better to use primary cells to study. Due to limited conditions, these experiments will also be carried out in future study.
This study studied the effects of madagascine on both endothelium and VSM because endothelium and VSM play different pathophysiological roles in vascular diseases (Santulli et al., 2011, 2014). For example, the lack of discrimination between proliferating VSM cells and endothelial cells may increase the risk of late thrombosis following angioplasty (Santulli et al., 2014). Madagascine-induced vasodilatation was transient and this transient vasodilatation effect on blood pressure in vivo needs further study. The highest concentration of madagascine used in this study is $100 \mu \mathrm{M}$ and it was shown that madagascine and its parent compound emodin shows almost no cell toxicity on normal cells but cancer cells (Epifano et al., 2013; Wei et al., 2013). The rat MRAs kept good condition after madagascine treatment by washing in this study, which also confirmed almost no cell toxicity of madagascine. However, the higher concentration of madagascine is also to be used to test its effects in vivo study. The aim of this study is to study the vasodilatory effect of madagascine on vasoconstriction in vitro, and to uncover weather AMPK activation is involved in madagascine induced vasodilatation. The other roles including potassium channel, alpha adrenergic receptors, and some vasodilatation factors in madagascine induced vasodilatation maybe, respectively, studied in future.

Cardiovascular diseases are often associated with co-occurring atherosclerosis, hypercholesterolemia, and inflammation, and madagascine induced activation of AMPK also is potentially beneficial for the prevention against and amelioration of the co-occurring disorders based on its modulation on VSM and circadian rhythm (Fan et al., 2011). Madagascine has potent biological activity and is safer as a potent AMPK activator. Madagascine-induced activation of AMPK leads to inhibition of vasoconstriction suggests its potential value in amelioration of vasospasm related cardiovascular diseases.

\section{AUTHOR CONTRIBUTIONS}

YL, DC, FE, SK, and PS designed the study. DC, BL, PS, SH, and DY performed the tissue research. BL, YX, YJ, and FT performed the cell research. YL, SG, and FE contributed reagents or analytic tools. DC, SK, and YX analyzed data. YL and DC wrote and revised the manuscript.

\section{FUNDING}

This study was supported by the National Natural Science Foundation of China (grant No. 30772601 and 81600440).

\section{ACKNOWLEDGMENT}

The authors wish to thank Lei Shi for their helpful discussions and reading of this manuscript. 


\section{REFERENCES}

Bradley, E. A, Eringa, E. C., Stehouwer, C. D., Korstjens, I., van Nieuw Amerongen, G. P., Musters, R., et al. (2010). Activation of AMP-activated protein kinase by 5 -aminoimidazole-4-carboxamide-1-beta-D-ribofuranoside in the muscle microcirculation increases nitric oxide synthesis and microvascular perfusion. Arterioscler. Thromb. Vasc. Biol. 30, 1137-1142. doi: 10.1161/ATVBAHA.110. 204404

Chen, D., Xiong, Y., Lin, Y., Tang, Z., Wang, J., Wang, L., et al. (2015). Capsaicin alleviates abnormal intestinal motility through regulation of enteric motor neurons and MLCK activity: relevance to intestinal motility disorders. Mol. Nutr. Food Res. 59, 1482-1490. doi: 10.1002/mnfr.201500039

Cox, R. H., and Rusch, N. J. (2002). New expression profiles of voltage-gated ion channels in arteries exposed to high blood pressure. Microcirculation 9, 243-257. doi: 10.1080/mic.9.4.243.257

Delle Monache, G., Delle Monache, F., Di Benedetto, R., and Oguakwa, J. U. (1987). New metabolites from Psorospermum tenuifolium. Phytochemistry 26, 2611-2613. doi: 10.1016/S0031-9422(00)83889-3

Ee, G. C. L., Sukari, M., Aspollah, M., Sim, W., and Lim, C. (2011). Cratoxylum glaucum and cratoxylum arborescens (Guttiferae)-two potential source of antioxidant agents. Asian J. Chem. 23, 569-572.

Epifano, F., Fiorito, S., Carlucci, G., Locatelli, M., and Genovese, S. (2013). Phytochemistry and pharmacognosy of naturally occurring prenyloxyanthraquinones. Curr. Drug Targets 14, 959-963. doi: 10.2174/138 9450111314090004

Fan, W., Downes, M., Atkins, A., Yu, R., and Evans, R. M. (2011). Nuclear receptors and AMPK: resetting metabolism. Cold. Spring Harb. Symp. Quant. Biol. 76, 17-22. doi: 10.1101/sqb.2012.76.010470

Fumiaki, N., Sei, K., Kimiko, M., Yoichi, M., Satoshi, S., Saori, M, et al. (2002). Involvement of Src family protein tyrosine kinases in $\mathrm{Ca}\left({ }^{2+}\right)$ sensitization of coronary artery contraction mediated by a sphingosylphosphorylcholineRho-kinase pathway. Circ. Res. 91, 953-960. doi: 10.1161/01.RES. 0000042702.04920.BF

Hisham, N. F., and Bayraktutan, U. (2012). Epidemiology, pathophysiology, and treatment of hypertension in ischaemic stroke patients. J. Stroke Cerebrovasc. Dis. 22: e4-14. doi: 10.1016/j.jstrokecerebrovasdis.2012. 05.001

Iinuma, M., Tosa, H., Ito, T., Tanaka,T., and Aqil, M. (1995). Two prenylated anthrones in Harungana madagascariensis. Phytochemistry 40, 267-270. doi: 10.1016/0031-9422(95)00202-I

Ji, L. (2013). Association between polymorphisms in the renin-angiotensinaldosterone system genes and essential hypertension in the han Chinese population. PLoS ONE 8:e72701. doi: 10.1371/journal.pone.0072701

Kaplun, L., Ivantsiv, Y., Bakhrat, A., and Raveh, D. (2003). DNA damage responsemediated degradation of Ho endonuclease via the ubiquitin system involves its nuclear export. J. Biol. Chem. 278, 48727-48734. doi: 10.1074/jbc.M30867 1200

Kondo, K., Okuno, T., Konishi, K., Saruta, T., and Kato, E. (1979). Central and peripheral effects of bradykinin and prostaglandin E2 on blood pressure in conscious rats. Naunyn Schmiedebergs Arch. Pharmacol. 308, 111-115. doi: 10.1007/BF00499052

Kumar, P., Mastan, K., Chowdhary, R., and Shanmugam, K. (2012). Oral manifestations in hypertensive patients: a clinical study. J. Oral Maxillofac. Pathol. 16, 215-221. doi: 10.4103/0973-029X.99069

Machino, T., Murakoshi, N., Sato, A., Xu, D. Z., Hoshi, T., Kimura T, et al. (2014). Anti-hypertensive effect of radiofrequency renal denervation in spontaneously hypertensive rats. Life Sci. 110, 86-92. doi: 10.1016/j.lfs.2014. 06.015

Majithiya, J. B., and Balaraman, R. (2006). Metformin reduces blood pressure and restores endothelial function in aorta of streptozotocin-induced diabetic rats. Life Sci. 78, 2615-2624. doi: 10.1016/j.lfs.2005.10.020

Mariman, E. C. M., and Wang, P. (2010). Adipocyte extracellular matrix composition, dynamics and role in obesity. Cell. Mol. Life Sci. 67, 1277-1292. doi: 10.1007/s00018-010-0263-4

Mbaveng, A. T., Kuete, V., Nguemeving, J. R., Beng, V. P., Nkengfack, A. E., Meyer, J. M., et al. (2008). Antimicrobial activity of the extracts and compounds obtained from Vismia guineensis (Guttiferae). Asian J. Trad. Med. 3, 211-223.
Noriyasu, M., Hiroko, K., Masafumi, S., Fengling, G., Satoshi, S., Takashi, Y., et al. (2006). Cholesterol primes vascular smooth muscle to induce $\mathrm{Ca} 2$ sensitization mediated by a sphingosylphosphorylcholine-Rho-kinase pathway: possible role for membrane raft. Circ. Res. 99, 299-306. doi: 10.1161/01.RES.0000235877. 33682.e9

Reihill, J. A., Ewart, M. A., Hardie, D. G., and Salt, I. P. (2007). AMPactivated protein kinase mediates VEGF-stimulated endothelial NO production. Biochem. Biophys. Res. Commun. 354, 1084-1088. doi: 10.1016/ j.bbrc.2007.01.110

Ritchie, E., and Taylor, W. (1964). The constituents of Harungana madagascariensis Poir. Tetrahedron Lett. 5, 1431-1436. doi: 10.1016/j.jep.2016. 06.005

Salihi, D. (2013). Endothelium derived relaxation factors reduce sulfur dioxideinduced aortic relaxation. Open J. Mol. Integr. Physiol. 3, 181-185. doi: 10.4236/ ojmip.2013.34023

Santulli, G., Basilicata, M. F., De Simone, M., Del Giudice, C., Anastasio A, Sorriento, D., et al. (2011). Evaluation of the anti-angiogenic properties of the new selective $\alpha \mathrm{V} \beta 3$ integrin antagonist RGDechiHCit. J. Transl. Med. 9, 1-10. doi: 10.1186/1479-5876-9-7

Santulli, G., Wronska, A., Uryu, K., Diacovo, T. G., Gao, M., Marx, S. O., et al. (2014). A selective microRNA-based strategy inhibits restenosis while preserving endothelial function. J. Clin. Invest. 124, 4102-4114. doi: 10.1172/ JCI76069

Satoshi, S., Shiro, K., Masafumi, S., Saori, M., Fumiaki, N., Tetsu, K., et al. (2002). Sphingosylphosphorylcholine is a novel messenger for Rho-kinasemediated $\mathrm{Ca}^{2+}$ sensitization in the bovine cerebral artery: unimportant role for protein kinase C. Circ. Res. 91, 112-119. doi: 10.1161/01.RES.0000026057.13 161.42

Shiraki, H., Kawasaki, H., Tezuka, S., Nakatsuma, A., and Kurosaki, Y. (2000). Endogenous calcitonin gene-related peptide (CGRP) mediates adrenergicdependent vasodilation induced by nicotine in mesenteric resistance arteries of the rat. Br. J. Pharmacol. 130, 1083-1091. doi: 10.1038/sj.bjp.07 03376

Shuangxi, W., Bin, L., Benoit, V., and Ming-Hui, Z. (2011). Inhibition of the AMP-activated protein kinase- $\alpha 2$ accentuates agonist-induced vascular smooth muscle contraction and high blood pressure in mice. Hypertension 57, 1010 1017. doi: 10.1161/HYPERTENSIONAHA.110.168906

Somlyo, A. P., and Somlyo, A. V. (1994). Signal transduction and regulation in smooth muscle. Nature 372, 231-236. doi: 10.1038/372 $812 \mathrm{a} 0$

Somlyo, A. V. (2002). New roads leading to Ca2+ sensitization. Circ. Res. 91, 83-84. doi: 10.1161/01.RES.0000028341.63905.91

Song, P., Kim, J. H., Ghim, J., Yoon, J. H., Lee, A., Kwon, Y., et al. (2013). Emodin regulates glucose utilization by activating AMP-activated protein kinase. J. Biol. Chem. 288, 5732-5742. doi: 10.1074/jbc.M112.441477

Stahmann, N., Woods, A., Carling, D., and Heller, R. (2006). Thrombin activates AMP-activated protein kinase in endothelial cells via a pathway involving Ca2+/calmodulin-dependent protein kinase beta. Mol. Cell. Biol. 26, 59335945. doi: 10.1128/MCB.00383-06

Subramaniam, A., Shanmugam, M. K., Ong, T. H., Li, F., Perumal, E., Chen, L., et al. (2013). Emodin inhibits growth and induces apoptosis in an orthotopic hepatocellular carcinoma model by blocking activation of STAT3. Br. J. Pharmacol. 170, 807-821. doi: 10.1111/bph.12302

Sun, P., Jin, X., Koyama, T., Li, S., Kitamura, Y., and Kawasaki, H. (2009). R-(-)alpha-methylhistamine, a histamine $\mathrm{H} 3$ receptor agonist, induces endotheliumdependent vasodilation in rat mesenteric resistance arteries. Biol. Pharm. Bull. 33, 58-63. doi: 10.1248/bpb.33.58

Todoroki-Ikeda, N., Mizukami, Y., Mogami, K., Kusuda, T., Yamamoto, K., Miyake, T., et al. (2000). Sphingosylphosphorylcholine induces $\mathrm{Ca}^{2+}$ sensitization of vascular smooth muscle contraction: possible involvement of Rho-kinase. FEBS Lett. 482, 85-90. doi: 10.1016/S0014-5793(00)0 2046-9

Wang, S., Liang, B., Viollet, B., and Zou, M. H. (2011). Inhibition of the AMP-activated protein kinase alpha 2 accentuates agonist-induced vascular smooth muscle contraction and high blood pressure in mice. Hypertension 57, 1010-1017. doi: 10.1161/HYPERTENSIONAHA.110.16 8906 
Wei, R., Ma, S., Wang, C., Ke, J., Yang, J., Li, W., et al. (2016). Exenatide exerts direct protective effects on endothelial cells through AMPK/Akt/eNOS pathway in a GLP-1 receptor dependent manner. Am. J. Physiol. Endocrinol. Metab. 310:E947-57. doi: 10.1152/ajpendo.00400. 2015

Wei, W. T., Lin, S. Z., Liu, D. L., and Wang Z. H. (2013). The distinct mechanisms of the antitumor activity of emodin in different types of cancer (Review). Oncol. Rep. 30, 2555-2562. doi: 10.3892/or.2013. 2741

Xu, Q., Cao, K., Zhou, X. H., Wang, X. J., Wang, Y. F., and Chao, D. U. (2012). Effects of paeonol on expression of eNOS and level of NO in HUVECs injured by homocysteine. Chin. Tradit. Patent. Med. 34, 2286-2289.

Yang, J. H., Wang, N., Jing-Yan, L. I., Zhang, J. J., and Feng, P. (2009). Effects of resveratrol on SIRT1 expression, eNOS activity and NO secretion of hyperlipid cultured HUVEC. China Pharm. 20, 981-983.
Yue, H. W., Jing, Q. C., Liu, P. P., Liu, J., Li, W. J., and Zhao, J. (2015). Sphingosylphosphorylcholine in cancer progress. Int. J. Clin. Exp. Med. 8, 11913-11921.

Conflict of Interest Statement: The authors declare that the research was conducted in the absence of any commercial or financial relationships that could be construed as a potential conflict of interest.

Copyright (c) 2016 Chen, Lv, Kobayashi, Xiong, Sun, Lin, Genovese, Epifano, Hou, Tang, Ji and $Y u$. This is an open-access article distributed under the terms of the Creative Commons Attribution License (CC BY). The use, distribution or reproduction in other forums is permitted, provided the original author (s) or licensor are credited and that the original publication in this journal is cited, in accordance with accepted academic practice. No use, distribution or reproduction is permitted which does not comply with these terms. 